\title{
Electrophysiology - Initial Sri Lankan Experience
}

\section{Dunuwille AN, Gunawardane $R$,}

Electrophysiology services, though established throughout the world for the diagnosis and treatment of arrhythmias, were unavailable in Sri Lanka until recently. The first Electrophysiology laboratory was set up in August 2003 at the Institute of Cardiology at the National Hospital of Sri Lanka. This is the main cardiology referral center for the whole country. Here we report our initial experience in electrophysiology during this short period of six months. Our lab consists of a Bard Duo analyzer with a Micropace stimulator and an EPT 1000 RF ablator. The team consists of a Consultant Cardiac Electrophysiologist, a Senior Registrar, two ECG technicians and an assisting nurse, Only the Consultant has undergone formal training in EP. The ECG technicians are trained in monitoring for vascular interventions and one has undergone a short (two week) training course in handling the EP lab. All others in the team receive on the job training. All studies have been done using a total of 25 Bard EP catheters ( 5 ablation and the rest a selection of quad and decapolar diagnostic catheters) that have been re-used after gas sterilization. Our lab time consists of one day a week. The total number of days available since the inception is 17 days.

As this was the initial experience in EP for most of the staff involved, we confined ourselves to one ablation and a few diagnostic studies per session. During this period we have performed 24 diagnostic studies and 19 ablations. The diagnostic studies consist of Bradycardia, Tachycardia and VT stimulation studies. We have performed ablations for AV Junctional re-entry tachycardia, (AVJRT), accessory pathways, atrial flutter as well as AV node ablations. We have not had any complications and the success of the ablations performed is encouraging. At present a separate cath lab for EP laboratory is been set up and this will be one of the few dedicated EP labs in the region. We hope, with the growth of our facilities and experience of our staff, we will be able to provide a meaningful service for patients with arrhythmias in this country. 